\title{
Processo de envelhecimento do idoso e a protagonização do enfermeiro na visita domiciliar na atenção primária de saúde
}

\author{
Aging process of the elderly and the role of nurses in home visits in primary health care \\ Proceso de envejecimento de los ancianos y el papel de las enfermeras en las visitas domiciliarias en \\ la atención primaria de salud
}

\section{Wanderson Alves Ribeiro ${ }^{1}$, Bruna Porath Azevedo Fassarella ${ }^{2}$, Keila do Carmo Neves ${ }^{3}$, Juliana de Lima Gomes ${ }^{4}$, Roberta Gomes Santos Oliveira ${ }^{5}$, Rafael Luiz Amorim de Oliveira ${ }^{6}$}

Como citar esse artigo. Ribeiro, WA; Fassarella, BPA; Neves, KC; Gomes, JL; Oliveira, RGS; Oliveira, RLA. Processo de envelhecimento do idoso e a protagonização do enfermeiro na visita domiciliar na atenção primária de saúde. Revista Pró-UniverSUS. 2019 Jul./Dez.; 10 (2): 53-58

\section{Resumo}

Trata-se de uma pesquisa bibliográfica de abordagem qualitativa e caráter descritivo que tem como objeto de estudo o protagonismo do médico de família na visita domiciliar, que tem como objetivo descrever o processo de envelhecimento do idoso e a protagonização do enfermeiro na visita domiciliar na atenção primária de saúde. O aumento da população idosa é uma realidade de diversos países, principalmente nos mais desenvolvidos, sendo assim existe uma grande preocupação em preservar a saúde e o bem-estar global para atender as especificidades desta etapa de vida. Como metodologia, utilizou-se a Biblioteca Virtual de Saúde, nas bases de informações LILACS, BDENF, MEDLINE e SCIELO, com recorte temporal de 2008 à 2018. É necessário que os enfermeiros venham desenvolver conhecimentos técnicos especializados referentes ao idosos. Conclui-se que entende-se que o enfermeiro tem papel de grande relevância, como educador, no processo de envelhecimento do idoso e sua família.

Palavras-chave: Envelhecimento, Enfermagem, Visita Domiciliar.

\begin{abstract}
Objectives: This is a bibliographical research of qualitative approach and descriptive character that has as object of study the protagonism of the family doctor in the home visit, which aims to describe the aging process of the elderly and the role of the nurse in the home visit in the care.primary health care. The increase in the elderly population is a reality of many countries, especially in the most developed, so there is a great concern to preserve health and global well-being to meet the specificities of this stage of life. As methodology, we used the Virtual Health Library, in the databases LILACS, BDENF, MEDLINE and SCIELO, with time frame from 2008 to 2018. It is necessary that nurses come to develop specialized technical knowledge regarding the elderly. It is concluded that it is understood that the nurse has a very important role as an educator in the aging process of the elderly and their families.
\end{abstract}

Keywords: Aging, Nursing, Home visit.

\section{Resumen}

Objetivos: Esta es una investigación bibliográfica de enfoque cualitativo y de carácter descriptivo que tiene como objeto de estudio el protagonismo del médico de familia en la visita domiciliaria, cuyo objetivo es describir el proceso de envejecimiento de los ancianos y el papel de la enfermera en la visita domiciliaria en la atención. Atención primaria de salud. El aumento de la población de adultos mayores es una realidad en muchos países, especialmente en los más desarrollados, por lo que existe una gran preocupación por preservar la salud y el bienestar global para satisfacer las especificidades de esta etapa de la vida. Como metodología, utilizamos la Biblioteca Virtual de Salud, en las bases de datos LILACS, BDENF, MEDLINE y SCIELO, con un período de tiempo de 2008 a 2018. Es necesario que las enfermeras vengan a desarrollar conocimientos técnicos especializados con respecto a los ancianos. Se concluye que se entiende que la enfermera tiene un papel muy importante como educador en el proceso de envejecimiento de los ancianos y sus familias.

Palabras clave: Envejecimiento Enfermería, Visita al hogar.

\footnotetext{
Afiliação dos autores: 1. Enfermeiro. Mestre pelo Programa Acadêmico em Ciências do Cuidado em Saúde, UFF, RJ, Brasil. Email: nursing_war@hotmail.com ORCID: https:// orcid.org/0000-0001-8655-3789

2. Enfermeira. Mestranda em Ciências Aplicadas em Saúde da Universidade de Vassouras. Docente do Curso de Graduação da UNIG. RJ, Brasil. Email: brunaporath@gmail.com ORCID: https://orcid.org/0000-0002-1400-4147

3. Enfermeira. Doutora em Enfermagem pela UFRJ. Docente do Curso de Graduação em Enfermagem da UNIG, UFF, RJ, Brasil. Email: keila arcanjo@hotmail.com ORCID: https://orcid.org/0000-0001-6164-1336

4. Enfermeira. Graduada pela Universidade Iguaçu, RJ, Brasil. Email: juliana.limag@hotmail.com ORCID: https://orcid.org/0000-0002-4907-1710

5. Enfermeira. Graduada pela Universidade Iguaçu, RJ, Brasil. Email: roberta.enferm93@hotmail.com ORCID: https://orcid.org/0000-0003-0076-1507

6 Acadêmico do Curso de Graduação em Enfermagem da Escola de Enfermagem Anna Nery/UFRJ. RJ, Brasil. Email: rafaelluiz.a.m@gmail.com ORCID: https://orcid.org/0000-

0003-3028-0421
} 


\section{Introdução}

De acordo com o Instituto Brasileiro de Geografia e Estatística (IBGE), a população de 60 anos ou mais no país corresponde a 30,2 milhões em 2017 da população total. $\mathrm{O}$ aumento da população idosa é uma realidade de diversos países, principalmente nos mais desenvolvidos, sendo assim existe uma grande preocupação em preservar a saúde e o bem-estar global para atender as especificidades desta etapa de vida, o idoso tem merecido atenção especial, pois o processo de envelhecer saudável implica cuidados de promoção, prevenção, educação, intervenção. Requer envolvimento e qualificação dos profissionais da atenção básica, com abordagem multiprofissional e interdisciplinar. ${ }^{1}$

Nesse sentido, menciona-se que o reconhecimento da magnitude do envelhecimento populacional no Brasil, em 4 de janeiro de 1994 foi aprovada a Lei $\mathrm{N}^{\circ} 8.842 / 1994$, que estabelece a Política Nacional do Idoso, posteriormente regulamentada pelo Decreto $\mathrm{N}^{\mathrm{o}} 1.948 / 96.6$ Esta Lei tem por finalidade assegurar direitos sociais que garantam a promoção da autonomia, integração e participação efetiva do idoso na sociedade, de modo a exercer sua cidadania. ${ }^{2}$

$\mathrm{O}$ aumento seguido em grande escala desta população pede uma revisão das políticas de saúde, onde há uma primordialidade na promoção do envelhecimento saudável e ativo, prevenindo assim os agravos ao longo de todo o ciclo vital, atendendo as necessidades com especificidade para com esta população. ${ }^{3}$

De acordo com o Sistema Único de Saúde (SUS) a finalidade primordial da política nacional de saúde da pessoa idosa é recuperar, manter e promover a autonomia e a independência dos idosos, obtendo medidas sendo em grupo ou individual de saúde, é considerado idoso e alvo dessas políticas todo homem ou mulher que possui 60 anos ou mais. ${ }^{4}$

Há uma dificuldade do governo em ampliar as propostas relacionadas à política do idoso, mesmo sabendo o quanto é muito necessário, devido ao envelhecimento da população. Os serviços de atenção básica são eficientes para promover uma assistência de qualidade ao idoso. ${ }^{5}$

Cabe mencionar que, é de grande relevância promover a autonomia dos idosos, respeitando o que lhe é de direito, como sua capacidade de se autodeterminar, mantendo preservado sua dignidade, sua integralidade e sua liberdade de escolha, no que é fundamental para promover sua qualidade de vida. ${ }^{6}$

Subsidiando o supracitado, a visita domiciliar (VD) é considerada como um meio importante de interação entre a Estratégia Saúde da Família (ESF) e as famílias, facilitando o acesso aos serviços, à construção de novas relações entre os usuários, a equipe e a formação de vínculo entre estes. Contribuindo para a melhoria das ações no tratamento e atenção aos idosos no processo saúde/doença, é através da visita domiciliar que se realiza atividades humanas e acolhedoras criando laços de confiança ao idoso no seu ambiente familiar seja ela realizada por Agentes Comunitário de Saúde (ACS), enfermeiros, médicos. ${ }^{6}$

No que se referente ao que é escrito nas políticas de saúde ao idoso, os objetivos são manter ao máximo o idoso na vivência com sua família e a comunidade, seu deslocamento para um serviço de longa permanência: hospital, asilo ou similar, deve ser a última alternativa. ${ }^{7}$

Corroborando ao contexto, vale informar que, a visita domiciliar é uma maneira de contribuir para um melhor desenvolvimento na assistência prestada, é um instrumento utilizado pelas equipes para inserção e conhecimento do contexto de vida da população, assim como estabelecimento de vínculos entre profissionais e usuários, passando a agir ao invés de aguardar as pessoas enfermas, e identificar explorar recursos e atuar em torno identificando necessidades e garantindo saúde e cuidado necessário, passando a entender e conceituara visita domiciliar como um foco que atravessa o sistema de saúde do Brasil. ${ }^{8}$

Pode-se dizer ainda que, a visita domiciliar é uma opção de cuidado que traz benefícios especialmente para os idosos com doenças incapacitantes e aqueles que dependem do auxílio de terceiros por longo período de tempo. Essas pessoas, muitas vezes, não dispõem de um indivíduo que se responsabilize por seus cuidados diários. ${ }^{9}$

Nos cuidados domiciliários, o enfermeiro entra no espaço cultural e vivencial de cada idoso, o que exige uma grande capacidade de adequação, mas também de entendimento das diferentes particularidades de cada um desses espaços no sentido de conquistar a confiança das pessoas cuidadas. De acordo com a teoria geral de Orem, os enfermeiros são designados como agentes de autocuidado terapêutico,quando as pessoas, por si só, não têm capacidade de desenvolver o autocuidado, entra o trabalho do enfermeiro no processo de cuidar. ${ }^{10}$

Referente a Visita Domiciliar (VD), pode ser definido como um dos instrumentos mais indicados na prestação de cuidados a saúde do idoso, se caracteriza de uma forma leve, a ideia primordial é a continuação do processo de trabalho contribua para criar situações próximas da realidade na qual este usuário está inserido, de modo que estimule reflexões acerca da necessidade de ressignificar práticas, valores e atitudes. ${ }^{11}$

Através dessas visitas é verificado as medidas antropométricas, fornecem informações a respeito da alimentação saudável, prática de exercícios físicos, e a prevenção das doenças diabéticas e hipertensas ou até mesmo as complicações. As ações desenvolvidas no domicílio privilegiam tanto ações de promoção quanto de recuperação da saúde do idoso e da família. ${ }^{11}$

Vale informar que é pertinente o cuidado 
individual para cada idoso, sendo assim reconhece a singularidade, valores, e não deixando de identificar suas características pessoais, condições clínicas, participação do autocuidado, impacta positivamente no resultado da assistência. $^{12}$

Diante disso, necessário reconhecer os fatores que influenciam o envelhecimento, relacionados com a biologia humana, como as capacidades funcionais, físicas, mentais e sociais, e respectiva capacidade de adaptação aos elementos ambientais, como os estilos de vida, influenciados pela informação a que se tem acesso, bem como os comportamentos que foram adquirindo e integrando no cotidiano. ${ }^{13}$

A enfermagem necessita buscar o aprimoramento quanto à assistência, buscando um cuidado individual $\mathrm{e}$ personalizado para que exista um profissional com esse pensamento ampliado, dominando todos os programas de assistência à saúde na rede básica por ser uma área de primeiro contato à saúde. É necessário incluir na formação acadêmica uma metodologia problematize e que faça com que o acadêmico de enfermagem seja capaz de propor e executar mudanças no modelo de cuidar se tornando um enfermeiro crítico não só no âmbito hospital, mas também na rede básica de saúde, onde o cuidado é essencial, pois evita agravos à saúde e dar uma atenção ao idoso são uma tática de promover uma saúde para nossa população aumentando a qualidade de vida. $^{7}$

Diante da problemática apresentada podese destacar como objeto de estudo o processo de envelhecimento do idoso.

Para tal, traçou-se a seguinte questão norteadora: Qual a protagonização do enfermeiro na visita domiciliar na atenção primária de saúde frente ao processo de envelhecimento do idoso?

Diante disso, a pesquisa tem como objetivo descrever o processo de envelhecimento do idoso e a protagonização do enfermeiro na visita domiciliar na atenção primária de saúde.

\section{Metodologia}

Entende - se por metodologia todas as ciências caracterizam-se pela utilização de métodos científicos; em contrapartida, nem todos os ramos de estudo que empregam estes métodos são ciências. Dessas afirmações podemos concluir que a utilização de métodos científicos não é da alçada exclusiva da ciência, mas não há ciência sem o emprego de métodos científicos. ${ }^{13}$

Trata-se de uma pesquisa bibliográfica de abordagem qualitativa e caráter descritivo. Cabe ressaltar que a pesquisa bibliográfica que é desenvolvida com auxílio de material já elaborado, constituído principalmente de livros e artigos científicos. Contudo em grande parte dos estudos seja exigido algum tipo de trabalho deste gênero, há pesquisas desenvolvidas exclusivamente a partir de fontes bibliográficas. ${ }^{8}$

Em relação ao método qualitativo, discorre que é o processo aplicado ao estudo da biografia, das representações e classificações que os seres humanos fazem a respeito de como vivem, edificam seus componentes e a si mesmos, sentem e pensam. ${ }^{14}$

De acordo as pesquisas descritivas possuem como objetivo a descrição das características de uma população, fenômeno ou de uma experiência. ${ }^{14}$

Os dados foram coletados em base de dados virtuais. Para tal utilizou-se a Biblioteca Virtual de Saúde (BVS), na seguinte base de informação: Literatura Latino-Americana e do Caribe em Ciências da Saúde (LILACS), Literatura Internacional em Ciência da Saúde (MEDLINE), Scientific Eletronic Library Online (SCIELO), dentre outros, no período de Abril à Junho de 2019.

Optou-se pelos seguintes descritores: Envelhecimento; Enfermagem e Visita Domiciliar que se encontram nos Descritores em Ciência da Saúde (DECS).

Estabeleceu-se então para a realização da pesquisa os critérios de inclusão: textos na íntegra, artigos científicos e em português com abordagem da temática estabelecida e que obedecessem ao recorte temporal de 2008 a 2018 e como critérios de exclusão, os textos incompletos, dissertações, teses e em língua estrangeira, textos que não abordassem a temática estabelecida e com recorte temporal inferior a 2008.

Cabe mencionar que os textos em língua estrangeira foram excluídos devido o interesse em embasar o estudo com dados do panorama brasileiro e os textos incompletos, para oferecer melhor compreensão através da leitura de textos na integra.

Após a associação de todos os descritores foram encontrados 14 artigos, excluídos 05 e selecionados apenas 09 .

Com vista a ampliar o conhecimento, a recorrência e o estado da temática, foi realizada uma pesquisa bibliográfica no endereço eletrônico scholar. google.com.br, para embasamento e contextualização do tema em questão, onde foram aplicados os critérios de inclusão e exclusão e selecionados 04 artigos.

\section{Analise de dados e discussão de resultados}

Subsequente a seleção dos artigos, foi realizado uma leitura reflexiva dos trezes artigos, emergiu duas categorias: Processo de Envelhecimento do Idoso e A Protagonização do Enfermeiro na Visita Domiciliar ao Idoso. 


\section{$1{ }^{\text {a }}$ Categoria: Processo de Envelhecimento do Idoso}

Envelhecer é um processo que é de acordo com cada pessoa, cada indivíduo tem reações diferentes, uns são bem mais rápidos, já em outros é gradativamente. Todo processo depende de muitos fatores como: estilo de vida, doenças crônicas. ${ }^{18}$

Com base no grande número de idosos no Brasil e a incidência de vários problemas relacionados a saúde desta população, é necessário prover maior acesso aos serviços de saúde e a cuidados de enfermagem nas áreas ambulatorial, hospitalar, comunitária e domiciliar. ${ }^{19}$

Dar um período para iniciar a velhice é uma tarefa nada fácil, pois é muito complicado, é difícil generalizarmos em se tratando de velhice, há características expressivas no meio de variados perfis de idosos e velhice, compreendemos que a idade é um caso definido, porém as intervenções que os idosos recebem aos anos estão sujeito à particularidade de cada pessoa. ${ }^{20}$

Há formas variadas de se definir a velhice e uma delas, é a explicação prescrita pela (OMS) Organização Mundial da Saúde, que é respaldada na idade cronológica, onde o conceito para idoso começa a partir dos 65 anos, isso nos países em desenvolvimento. Já no Brasil, consoante com o estatuto do idoso, pessoas com idade de 60 anos ou acima dos 60 , são aceitas como idosas. $^{3}$

Nesse sentido, cabe mencionar que envelhecimento é universal, constitui-se em um estágio de desenvolvimento humano, assim como nas outras etapas da vida, a sociedade vivencia conjuntos de perdas e ganhos. $\mathrm{O}$ corpo talvez já não tenha viço da juventude, a disposição não é mais a mesma, e as atividades da vida diárias se tornam mais lentas, é de suma importância que o idoso mantenha sua autonomia, porém irá agregar muito em sua vida física e mental ter em sua rotina funções a serem realizadas, sendo assim fará eles se sentirem úteis, onde muitas das vezes são impedidas pelos familiares com motivos de que não são capazes pela idade avançada. ${ }^{19}$

Temos também o conceito biológico em que sua reaçãoé pelo ângulo celular, molecular,orgânica, tecidual do indivíduo, ao passo que pelo ponto de vista psíquico é a conexão existente entre as dimensões cognitiva e psicoafetivas, modificando o desenvolvimento da personalidade e afeto. De certa forma, se expressar sobre o envelhecimento é estabelecer várias interpretações que se misturam aos atos habituais e nas várias formas culturais. ${ }^{6}$

Ressalta-se que, de forma inevitável o ser humano está continuamente, a pensar e ter preocupações com o envelhecimento e vendo de diversas formas, e contraindo uma extensão heterogênea. Conforme as projeções da OMS, os resultados mostrados, revelaram um acréscimo, um tanto quanto alto. ${ }^{19}$

Vale destacar que o envelhecimento é um acontecimento que atinge todo ser humano, independente de quem seja caracterizando por se alterar de modo continuo evoluindo progressivamente sendo irreversível e que está relacionado à vários elementos que contribuem e influenciam no processo de envelhecimento, que são os fatores biológico, sociológico e psicológico. ${ }^{19}$

O propósito de ter uma vida intensa é a intenção de qualquer ser humano. Mas,contudo, esse avanço acontece de acordo que o indivíduo tenha uma vida com mais qualidade. Dando assim, todo e qualquer projeto direcionado ao idoso, deve ser pensado na competência funcional na autonomia que é necessária, na autossatisfação. Precisamos também entender que o idoso necessita de estarem inseridos nos mais variados contextos sociais e de dar um novo direcionamento, um novo significado, um valor, além do que se tem para a vida na idade avançada, e incitar primordialmente os cuidados a prevenção e dar a atenção absoluta a saúde. ${ }^{5}$

Todo ser humano,toda espécie envelhececom o passar dos tempos e sofrem modificações desde o nascimento até a morte. Foram estabelecidas pelos cientistas, teorias na qual tentam nos fazer entender, qual o motivo das pessoas envelhecerem, se bem que nenhuma tenha obtido confirmação.

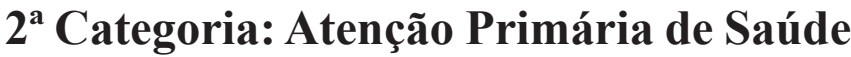 e aProtagonização do Enfermeiro na Visita Domiciliar ao Idoso.}

Decorrente de um curso sucedido há mais de duas décadasa Atenção Primária à Saúde (APS) é ratificada como a base de um sistema de saúde eficaz e responsivo. A Declaração de Alma-Ata de 1978 que reiterou o direito ao mais alto nível de saúde, com igualdade, solidariedade e o direito à saúde como seus valores primordiais. ${ }^{9}$

Salienta-sea necessidade de serviços de saúde abrangentes, não apenas curativos, mas também serviços que atendam às necessidades em termos de promoção da saúde, prevenção, reabilitação e tratamento de condições comuns. Um forte nível resolutivo de atenção primária de saúde é a base para o desenvolvimento de sistemas de saúde. ${ }^{20}$

Ao princípio do ano de 2008 o Relatório Mundial de Saúderestabeleceu em nível global a necessidade da abordagem da APS, fornecendo evidências concretas de que a mesma era acessível e tinha maior impacto na prestação de assistência de saúde onde as pessoas vivem. $^{21}$

Posteriormente no ano de 2014, os Estados Membros da Organização Pan-Americana da Saúde adotaram a Estratégia para o Acesso Universal à 
Saúde e Cobertura Universal de Saúde, que reitera o direito à saúde, solidariedade e equidade e promove o desenvolvimento de sistemas de saúde baseados na APS. $^{20}$

A Organização Pan-Americana da Saúde / Organização Mundial da Saúde (OPAS / OMS) apoia os países no estabelecimento de equipes interprofissionais de atenção primária à saúde, na transformação da educação em saúde e na capacitação no planejamento estratégico e gestão de recursos humanos para a saúde. ${ }^{5}$

O enfermeiro desempenha um papel crítico no avanço da APS. Novos perfis, novas práticasavançadas relacionadas e embasadas na melhoria do atendimento, sendo fundamentais nesse esforço e, em particular, na promoção da saúde, prevenção de doenças e cuidados, respeitando a particularidade de cada população. ${ }^{2}$

Reforça-se a Inclusão das ações do enfermeirono âmbito individual e coletivo, ampliandoa promoção e a proteção da saúde, a prevenção de agravos, o diagnóstico, o tratamento, a reabilitação, redução de danos e a manutenção da saúde, articulam-se por meio de ações intersetoriais no Brasil. ${ }^{7}$

O enfermeiro destaca-se nesse contexto de saúde, por algumas das suas atribuições específicas no tocante à prática clínica, quais sejam: realizar atenção à saúde aos indivíduos e famílias cadastradas nas equipes; realizar consulta de enfermagem, procedimentos, atividades em grupo conforme protocolos ou outras normativas técnicas estabelecidas pelo gestor federal, estadual, municipal ou do Distrito Federal, observadas as disposições legais da profissão; solicitar exames complementares; transcrição de medicações e encaminhar, quando necessário, usuários a outros serviços. ${ }^{7}$

$\mathrm{O}$ enfermeiroenquanto membro da equipe pode desenvolver atividades que também envolvem a clínica, de forma indireta, como: acolhimento, realização de visitas domiciliares, participação em reuniões de equipe a fim de planejar e avaliar as ações e participação em ações de educação em saúde. A prática clínica envolve, portanto, vários processos que são articulados e colaboram para que haja um cuidado integral ao cliente. ${ }^{7}$

É necessário que os enfermeiros venham desenvolver conhecimentos técnicos especializados referentes ao envelhecimento e ao cuidado domiciliário, para compreender as necessidades apresentadas pelos pacientes e propor um cuidado condizente a estes e conhecer a família em todas as suas dimensões, mantendo fortalecido o vínculo de confiança e proximidade entre seus membros e ao processo de cuidado. ${ }^{19}$

Vale destacar que a família tem se preocupado e se ocupado no cuidado de seus membros idosos. Por isso, é de suma importância que os profissionais enfermeiros estabeleçam e fortaleçam uma relação mais estreita de parceria com os familiares cuidadores, visualizando-os como colaboradores e clientes, uma vez que agregam hábitos de vida semelhantes aos dos idosos sob seus cuidados. ${ }^{9}$

De modo geral, com o seu saber teórico científico, o papel do enfermeiro é desenvolver atividades voltadas para o cuidado na visita domiciliar, este espaço favorece um cuidar que compreende mais que um momento tecnicista, mas uma atitude de envolvimento afetivo com o outro, fortalecendo o vínculo profissionalusuário, o enfermeiro tem a oportunidade de ouvir demandas, avaliar as condições de saúde físicas e psicoemocionais. ${ }^{20}$

A relação com os cuidadores é de extrema importância, age como um facilitador, para a identificação de problemas e possibilidades de auxílio ao usuário em seus modos de levar a vida, incentivando que expressem suas preocupações e experiências no cuidado ao paciente e buscando, ainda, o conhecer mais profundamente, assim como um "conselheiro", por ouvir e acolher famílias em relação a preocupações e medos. A visita permite ao profissional e sua equipe uma maior aproximação à realidade em que vive o indivíduo. ${ }^{21}$

A visita domiciliar exige preparo profissional, predisposição pessoal e disponibilidade de tempo na sua execução, por outro, é um serviço prestado dentro do próprio contexto, que parece agradar à maioria da população e pode diminuir a demanda pelas instituições de saúde, reduzindo custos para as famílias e o setor saúde. ${ }^{3}$

A atuação do enfermeiro na atenção primaria à saúde tem como propósito a realização das suas atividades baseado no conhecimento das ações a serem desenvolvidas, tendo que os profissionais carecem ter um olhar particular para todo cliente assistido. À vista disso, observa-se que é fundamental a aplicação de instrumentos que auxiliem o processo de assistencial no ambiente domiciliar, como a elaboração do vínculo por meio da boa dinâmica, buscando sempre aplicar a escuta e um bom acolhimento, visto isso realizando um atendimento e identificando os usuários com um todo $\mathrm{e}$ não focando exclusivamente sua patologia. ${ }^{15}$

Entende-se a importância do enfermeiro como educador na promoção da saúde, uma vez que é o profissional que possui conhecimentos técnicas e habilidades para realizar uma análise detalhada das condições do cliente idoso e do seu cuidador, atentar para as fragilidades e especificidades destes indivíduos, a fim de antecipar-se e intervir em possíveis complicações, para evitar que o estado de saúde piore e, assim, assegurar a qualidade de vida dos cliente. ${ }^{20}$

É por intermédio da visita que este profissional será apto de analisar as condições social e familiar em que vive o indivíduo, bem como fazer a busca ativa e idealizar e realizar as medidas assistenciais adequadas, com base na promoção da saúde. O enfermeiro realiza suas visitas domiciliar voltado não só pelo cliente, mas também para a sua à família. ${ }^{21}$ 


\section{Conclusão}

Conclui-se que a construção desse trabalho possibilitou um olhar mais profundo na atenção primaria à saúde e argumentar as estratégias promovidas na atenção do idoso, além dos idosos, ter uma percepção focada também no familiar, pois o índice de idosos no nosso país aumentou nos últimos anos e, nesse sentido, maior necessidade da idealização de estratégias de saúde organizadas pelo SUS, sendo preciso uma visão direcionada a grande população idosa, e a visita é a continuidade do cuidado, é uma ponte entre a clínica e as famílias que são cadastradas englobando e dissipando a saúde nas famílias nas áreas territoriais necessitadas.

Por fim, entende-se que o enfermeiro tem papel de grande relevância, como educador, no processo de envelhecimento do idoso e sua família, tento em vista que a educação em saúde poderá resgatar a autonomia deste idoso e ainda, contribuir para o processo de autocuidado do idoso e sua família.

\section{Referências}

1. Andrade AM, Silva KL, Seixas CT, Braga PP. Nursing practice in home care: an integrative literature review. RevBrasEnferm. 2017;70(1):210-217.

2. Basílio Rommel Almeida Fechine, Nicolino Tronpieri. O Processo do Envelhecimento: As principais alterações que acontecem com o idoso com o passar dos anos. Revista Científica Internacional, Edição 20(1)7.2012.

3. Brasil, Ministério da Saúde. Secretaria de Atenção Básica. Envelhecimento e saúde da pessoa idosa. Brasília: Ministério da Saúde;2006.

4. Brasil. Ministério da Saúde. Orientações técnicas para a implementação de linha de cuidado para atenção integral à saúde da pessoa idosa no sistema único de saúde. Brasília: março; 2018.

5. Brasil. Ministério da Saúde. Secretaria de Atenção à Saúde. Departamento de Atenção Básica. Política Nacional de Atenção Básica/Ministério da Saúde, Secretaria de Atenção à Saúde, Departamento de Atenção Básica. $4^{\mathrm{a}}$ ed. Brasília: Ministério da Saúde;2007.

6. CostaRHS, Couto CRO, Silva RAR. Prática clínica do enfermeiro na Estratégia de Saúde da Família. Rev. Enferm. Santa Maria.41(2).2015.

7. Gago AE, Lopes JM. Cuidados domiciliares: interação do enfermeiro com a pessoa idosa/família. 2012

8. GilAntonio Carlos. Como elaborar projetos de pesquisa. 5. ed. São Paulo: Atlas, 2008.

9. Gomes MFP,Fracolli, L.A, Machado BC. Atenção domiciliar do enfermeiro na Estratégia de Saúde da Família, 2015

10. Kawata LS, Mishima SM, Chirelli MQ et al. Os desempenhos da enfermeira na saúde da família- Construindo competência para o cuidado. Texto Contexto Enf, Florianópolis, 2013; 22(4): 961-70.

11. Kebian LVA, Acioli S. A visita Domiciliar de Enfermeiros e Agentes Comunitários de Saúde da Estratégia da Família. Rev. Elet. Enf.2014; 403408.

12. Kebian LVA, Acioli S. Visita Domiciliar: espaço de práticas de cuidado do enfermeiro e do agente comunitário de saúde. 2012

13. Lakatos EM, Marconi NA. Fundamentos de Metodologia Cientifica. 7. ed. São Paulo. Ed. Atlas, 2010.
14. Martins JJ, Schier J, Erdmann AL et al. Políticas públicas de atenção à saúde do idoso: reflexão acerca da capacitação dos profissionais da saúde para o cuidado com o idoso. RevBrasGeriatrGerontol.2017; 10(3).

15. Minayo Maria Cecília de Souza. O desafio do conhecimento: pesquisa qualitativa em saúde. In: $\mathrm{O}$ desafio do conhecimento: pesquisa qualitativa em saúde. 2014

16. Ministério da Saúde. Caderneta de saúde da pessoa idosa Brasília: Editora do Ministério da Saúde, 4 edição. 2017.

17. Nascimento JS, Costa LMC, Santos RM, et al. Visitas Domiciliares Como Estratégias de Promoção da Saúde pela Enfermagem. Rev. Bras. Promoc. Saúde, 2013; 26(4): 513-52

18. Silva, FAM. Ampliação do papel dos enfermeiros na atenção primária a saúde. 2018

19. Silvestre JA, Costa Neto MM. Abordagem do idoso em programas de saúde da família. Cad saúde pública.2003 mai/jun;19(3):839-47.

20. Sossai LC, Pinto IC. A visita domiciliária do enfermeiro: fragilidades $\mathrm{x}$ potencialidades. 2010.

21. Veras R. Em busca de uma assistência adequada à saúde do idoso:re visãodaliteraturaeaplicaçãodeuminstrumentode detecção precoce e de previsibilidade de agravos. Cad Saúde Pública. 2009; 19:705. 